\title{
Fetal lung fluid: Not the same as amniotic fluid
}

\author{
Hemananda Muniraman ${ }^{1}$ \\ ${ }^{1}$ Creighton University
}

December 12, 2020

\section{Fetal lung fluid: Not the same as amniotic fluid}

Author: Hemananda Muniraman MBBS, FAAP, FRCPCH

Affiliations:

Creighton University School of Medicine, Phoenix Campus, Arizona, US

Corresponding Author: Hemananda Muniraman, MBBS, FAAP, FRCPCH

Assistant Professor of Pediatrics Affiliate, Creighton University School of Medicine

Phoenix Campus, 350 W Thomas Rd, Phoenix, AZ, US 85013

Email; Hemu_Muniraman@mednax.com, Phone: +16022564628 Fax: +16026276325

Conflict of Interest Disclosure: I have no conflicts of interest relevant to this article to disclose. Funding/Support.

No funding to be reported

Key words: Extreme preterm, neonates, pneumatocele, fetal lung fluid

Dear Dr Murphy

Editor in chief, Pediatric Pulmonology

I read with interest the recently published article "Pulmonary pneumatoceles in neonates" by Dr Rocha. [1] It is a well written comprehensive review of pulmonary pneumatoceles in neonates and an important resource for the clinicians in decision making, that I found to be very insightful whilst recently managing an extreme preterm with a large pneumatocele.

However, I wanted to bring attention to a statement in the introduction where the author states that the "preterm infants' lungs are filled with amniotic fluid". This is not accurate and appears to be a common misconception, particularly among medical students and junior residents despite recognition of fetal lung fluid as being a separate entity from the amniotic fluid as originally described in 1948 by Jost and Policard. [2] I believe this is an important knowledge gap that needs to be addressed. Understanding of metabolism and role of fetal lung fluid in lung development and postnatal transition is essential to clinicians involved in the care of newborns and infants. Though a comprehensive review of fetal lung fluid is beyond the scope of this letter, I provide a brief basic overview of fetal lung fluid with references for more comprehensive reading.

Fetal lung fluid is a chloride rich acidic fluid produced with in the fetal lungs by secretion of chloride across the distal lung epithelial cells and is a major determinant for fetal lung growth and development. The fluid lung volume is maintained by transglottic pressure gradient with periodic egress of excess fluid during fetal breathing. Decreased lung fluid volume is associated with pulmonary hypoplasia and conversely, upper airway lesions obstructing the egress of lung fluid leads to increase in fetal lung fluid and volume, a principle 
used in fetal interventions such as endoluminal tracheal occlusion to enhance pulmonary growth in conditions namely congenital diaphragmatic hernia. $[2,3,4]$

As important the fetal lung fluid is for lung development, clearance of lung fluid is crucial for normal postnatal transition and establishment of air filled lungs for effective gas exchange. Clearance of lung fluid is a complex and coordinated process that starts before the process of birth itself. The rate of lung fluid secretion diminishes before labor at term gestation. Catecholamines namely epinephrine released during labor upregulates the epithelial sodium channels (ENaC) promoting influx of sodium and fluid from lumen into pulmonary interstitial space thereby reversing the direction of fluid movement in the perinatal period. Lung fluid is decreased to about $35 \%$ following active labor and birth which are further cleared with neonatal cry and breathing and inflow of air after birth. The fluid from the interstitial space is cleared over the next few hours via pulmonary circulation and lymphatic drainage. However this process is impaired in infants delivered by elective cesarean sections leading to increased retained lung fluid after birth and resulting in transient respiratory distress and tachypnea of newborn (TTN). In preterm infants, catecholamine induced fluid reabsorption via $\mathrm{ENaC}$ is limited and may contribute to respiratory distress after birth. Prenatal maternal steroids and triiodothyronine administration is known to induce expression of messenger RNA for ENaC subunits in the fetal lungs and may facilitate lung fluid clearance. $[2,4]$

Lastly, amniotic fluid aspiration into fetal lungs, with and without meconium contamination, has been reported to be the cause of respiratory distress with case reports of massive amniotic fluid aspiration noted on postmortem histological examination of newborn lungs. [5]

References:

Rocha G. Pulmonary pneumatoceles in neonates. Pediatr Pulmonol. 2020 Jul 21. doi: 10.1002/ppul.24969. Epub ahead of print. PMID: 32691976.

Katz C, Bentur L, Elias N. Clinical implication of lung fluid balance in the perinatal period. J Perinatol. 2011 Apr;31(4):230-5. doi: 10.1038/jp.2010.134. PMID: 21448181.

Hooper SB, Harding R. Fetal lung liquid: a major determinant of the growth and functional development of the fetal lung. Clin Exp Pharmacol Physiol. 1995 Apr;22(4):235-47. doi: 10.1111/j.14401681.1995.tb01988.x. PMID: 7671435

Kallapur S, Jobe A; Fetal Lung fluid. Richard J. Martin, Avroy A. Fanaroff, Michele C. Walsh. Fanaroff And Martin's Neonatal-Perinatal Medicine : Diseases of the Fetus and Infant, 10th edition. Philadelphia, PA :Elsevier/Saunders, 2015

Lavezzi AM, Poloniato A, Rovelli R, Lorioli L, Iasi GA, Pusiol T et al. Massive Amniotic Fluid Aspiration in a Case of Sudden Neonatal Death With Severe Hypoplasia of the Retrotrapezoid/Parafacial Respiratory Group. Front Pediatr. 2019 Apr 4;7:116. doi: 10.3389/fped.2019.00116. PMID: 31019904 